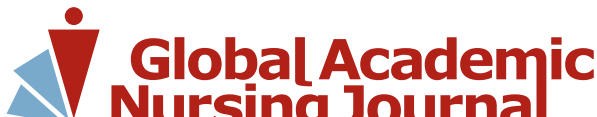 Nursing Journal
}

\section{Implementação de fluxo para manejo de teste rápido em unidade de saúde no Rio de Janeiro}

\author{
Flow implementation for rapid test management in a health facility in Rio de Janeiro
}

Implementación de flujo para la gestión rápida de pruebas en un establecimiento de salud en Río de Janeiro

\section{Bruna Caroline Hirle de Souza ${ }^{1}$ ORCID: 0000-0003-2259-8034 \\ Tatiana de Araujo Eleuterio ${ }^{2}$ ORCID: 0000-0002-8043-2350 Juliana Dias Cirilo ${ }^{3}$ ORCID: 0000-0002-0118-6375 Felipe Kaezer dos Santos ${ }^{2}$ ORCID: 0000-0002-2430-467X}

${ }^{1}$ Secretaria Municipal de Saúde. Rio de Janeiro, Brasil. 2Universidade do Estado do Rio de Janeiro. Rio de Janeiro, Brasil. ${ }^{3}$ Marinha do Brasil. Rio de Janeiro, Brasil.

\section{Como citar este artigo:}

Souza BCH, Eleuterio TA, Cirilo JD, Santos FK. Implementação de fluxo para manejo de teste rápido em unidade de saúde no Rio de Janeiro. Glob Acad Nurs. 2021;2(2):e142. https://dx.doi.org/10.5935/26755602.20200142

\section{Autor correspondente:}

Bruna Caroline Hirle de Souza E-mail:

brunacarolinehirle@gmail.com

Editor Chefe: Caroliny dos Santos Guimarães da Fonseca Editor Executivo: Kátia dos Santos Armada de Oliveira

Submissão: 29-03-2021 Aprovação: 27-08-2021

\section{Resumo}

Trata-se do relato de experiência de uma residente de enfermagem na implementação de um plano de intervenção voltado à resolução de um problema identificado em uma unidade de atenção primária do tipo $B$, onde coexistem o modelo de estratégia de saúde da família e o modelo tradicional, localizada na zona norte do município do Rio de Janeiro. O objetivo principal foi descrever a implementação de um fluxo para realização e registro de testes rápidos para HIV, sífilis, hepatites $\mathrm{B}$ e $\mathrm{C}$, visando a melhoria da qualidade da assistência durante a execução dos mesmos, a liberação de resultados por meio de formulários padronizados e a capacitação dos profissionais envolvidos. A metodologia se deu através de um plano de intervenção, onde o objetivo foi a implementação de um fluxograma para realização de testes rápidos para infecções sexualmente transmissíveis em uma unidade e capacitação da equipe que realizava os testes na unidade. A partir da implementação do fluxo, foram observadas melhorias no processo de realização e registro dos testes rápidos e também a manutenção de limitações referentes ao tema. Percebeu-se a falta de conhecimento dos profissionais envolvidos na testagem, com consequente impacto na eficácia, ressaltando-se a importância da educação continuada.

Descritores: Diagnóstico; Atenção Primária à Saúde; HIV; Hepatite; Sífilis; Enfermeiro.

\section{Abstract}

This is the experience report of a nursing resident in the implementation of an intervention plan aimed at solving a problem identified in a type B primary care unit, where the family health strategy model and the traditional model coexist, located in the northern part of the city of Rio de Janeiro. The aim was to describe the implementation of a flow for carrying out and recording rapid tests for HIV, syphilis, hepatitis B and C, aiming to improve the quality of care during their execution, the release of results through standardized forms and the training of the professionals involved. The methodology was carried out through an intervention plan, where the objective was to implement a flowchart for conducting rapid tests for sexually transmitted infections in a unit and training the team that performed the tests in the unit. From the implementation of the flow, improvements were observed in the process of carrying out and recording the quick tests, as well as the maintenance of limitations related to the topic. The lack of knowledge of the professionals involved in the testing was noticed, with a consequent impact on effectiveness, emphasizing the importance of continuing education.

Descriptors: Diagnosis; Primary Health Care; HIV; Hepatitis; Syphilis; Nurse.

\section{Resumén}

Este es el relato de experiencia de un residente de enfermería en la implementación de un plan de intervención orientado a la solución de un problema identificado en una unidad de atención primaria tipo $B$, donde conviven el modelo de estrategia de salud de la familia y el modelo tradicional, ubicado en la zona norte del país. ciudad de Rio de Janeiro. El objetivo principal fue describir la implementación de un flujo para la realización y registro de pruebas rápidas de $\mathrm{VIH}$, sífilis, hepatitis $\mathrm{B}$ y $\mathrm{C}$, con el objetivo de mejorar la calidad de la atención durante su ejecución, la divulgación de resultados a través de formularios estandarizados y la capacitación de los profesionales involucrados. La metodología se llevó a cabo a través de un plan de intervención, donde el objetivo fue implementar un diagrama de flujo para la realización de pruebas rápidas de infecciones de transmisión sexual en una unidad y capacitar al equipo que realizó las pruebas en la unidad. A partir de la implementación del flujo, se observaron mejoras en el proceso de realización y registro de las pruebas rápidas, así como el mantenimiento de limitaciones relacionadas con el tema. Se notó el desconocimiento de los profesionales involucrados en las pruebas, con el consecuente impacto en la efectividad, enfatizando la importancia de la educación continua.

Descriptores: Diagnóstico; Primeros Auxilios; HIV; Hepatitis; Sífilis; Enfermero. 


\section{Introdução}

As Infecções Sexualmente Transmissíveis (IST) são problemas de Saúde Pública devido à sua magnitude e dificuldade de acesso ao tratamento adequado ${ }^{1}$.

A Organização Mundial da Saúde (OMS) aponta em 2019, que a estimativa anual é de aproximadamente 376 milhões de novas infecções sexualmente transmissíveis².

A Síndrome da Imunodeficiência Adquirida (AIDS) foi identificada na década de 1980, difundindo-se e tornando-se uma pandemia. Tem como agente etiológico o vírus da imunodeficiência humana (HIV). O primeiro diagnóstico da doença no Brasil foi no município de São Paulo, em 1982. Os primeiros casos diagnosticados estavam relacionados a homens que faziam sexo com homens, pessoas em uso de drogas injetáveis, e receptores de transfusão sanguínea ${ }^{3}$. Nos anos de 1990 o termo vulnerabilidade surgiu para reafirmar que qualquer indivíduo poderia se infectar com o vírus do HIV4. Hoje, esse panorama segue se modificando, e atualmente há uma tendência de crescimento do número de casos entre jovens na faixa etária de 15 a 24 anos e nos adultos com mais de 50 anos, tanto em homens como em mulheres ${ }^{3}$.

Em 2012, os testes rápidos para diagnóstico do HIV, Sífilis e Hepatites Virais foram incluídos no programa da Rede Cegonha, contribuindo para o diagnóstico precoce no pré-natal, sendo essa uma estratégia fundamental para a redução da transmissão vertical ${ }^{5}$.

No modelo de organização do Sistema Único de Saúde (SUS), a Atenção Primária (AP) é a porta de entrada do usuário na rede. Desta forma, é ela a coordenadora do cuidado deste usuário, onde se realizará a testagem, diagnóstico e tratamento, com objetivo de aumentar o acesso ao serviço ${ }^{6}$. A Estratégia de Saúde da Família, enquanto unidade de atenção primária, se caracteriza por serviços de promoção, proteção da saúde e prevenção de agravos, tanto de forma individual como coletiva?.

O teste rápido é uma ferramenta que tem por objetivo atender às necessidades relacionadas à prevenção de HIV/AIDS e outras infecções sexualmente transmissíveis e à promoção da saúde ${ }^{8}$. O principal objetivo é oportunizar ao usuário um atendimento onde a equidade e a integralidade, princípios doutrinários do SUS, estejam presentes de forma universal para um diagnóstico precoce, consequente tratamento em tempo oportuno e quebra da cadeia de transmissão ${ }^{9}$. Além disso, o aconselhamento pré e pós teste é uma forma de educação em saúde, papel fundamental da Atenção Primária ${ }^{10}$. A descentralização da testagem rápida para as unidades de AP traz uma mudança significativa no atendimento, antes realizado prioritariamente em Centros Especializados de Testagem e Aconselhamento (CTAs) e, atualmente, disponível nas unidades mais próximas aos usuários, tornando-a um facilitador para captação precoce desses $\operatorname{casos}^{11}$.

Vale ressaltar o papel fundamental da consulta de enfermagem nesse processo; a Resolução do COFEN n.o $544 / 2017$ aborda a consulta como ato privativo do enfermeiro, na qual o profissional utiliza o método científico para identificar problemas no processo saúde/doença de um indivíduo, realizando a sistematização de enfermagem a fim de contribuir para a promoção, prevenção, proteção da saúde, recuperação e reabilitação do usuário ${ }^{12}$.

O presente estudo se trata de um relato de experiência, no qual o objetivo foi propor a implementação de um fluxo para realização e registro de testes rápidos para infecções sexualmente transmissíveis, sendo elas: HIV, sífilis, hepatites B e C, tendo em vista a melhoria da qualidade da assistência durante a execução dos mesmos, a liberação de resultados por meio de formulários padronizados e a capacitação dos profissionais envolvidos.

\section{Metodologia}

Um plano de intervenção surge em resposta a um problema concreto, daí afirmar que sua elaboração objetiva, antes de mais nada, deve contribuir para a solução de problemas, transformando ideias em ações, com o fim de alcançar objetivos específicos dentro dos limites de um orçamento e de um período de tempo. A elaboração de um plano é uma ferramenta gerencial que tem demonstrado crescente importância para a vida de toda a organização, independentemente da natureza do produto ou serviço oferecido pela mesma, ou de sua esfera de atuação pública ou privada, uma vez que visa a sistematização de ações e a otimização de atividades e processos, sejam eles estratégicos ou operacionais ${ }^{13}$.

Deste modo, o presente trabalho é um relato de experiência acerca da implementação de um plano de intervenção, que teve como ação proposta a melhoria no fluxo de testagem rápida para ISTs em uma unidade de Atenção Primária, situada na zona norte no município do Rio de Janeiro.

O presente plano de intervenção foi direcionado à toda unidade, na qual a autora atuava como enfermeira residente de enfermagem em saúde da família do segundo ano.

Através da vivência durante a residência no Centro Municipal de Saúde (CMS), foi identificada a ausência de um fluxo para armazenamento, manipulação, realização, registro e emissão de resultados dos testes rápidos para HIV, sífilis e hepatites B e C. Considerando a importância desse procedimento, entendeu-se que tal problema trazia a necessidade de uma intervenção para melhoria da qualidade do processo e criação de um fluxo único para toda a unidade.

O público-alvo deste plano de intervenção foram: as sete técnicas de enfermagem do CMS, os demais profissionais da equipe de saúde que prestam assistência aos usuários e que também eventualmente realizam testes rápidos e, indiretamente, todos os usuários que viessem a realizar a testagem rápida na unidade.

A partir da definição do problema, foram identificados os seguintes nós críticos: armazenamento incorreto dos kits dos testes rápidos, em não conformidade com a orientação do fabricante (temperatura máxima de 30 ㄷ); registro realizado nas planilhas manuais, com identificação e exposição dos pacientes, por meio de seus nomes e seus resultados; registro dos exames realizados com dados faltantes ou até mesmo não realização do registro; técnica de realização da coleta de forma incorreta ou com objetos não pertencentes aos respectivos kits; déficit 
de conhecimento dos profissionais quanto à importância das orientações preconizadas no manual; falta de certificação para realização dos testes rápidos pelo TELELAB, para todas as técnicas de enfermagem da unidade; não utilização dos formulários padrão para emissão de laudos de testes rápidos, disponíveis na plataforma SUBPAV.

\section{Desenho da Operação}

\section{1a etapa - Diagnóstico da Rotina}

Diante do problema definido, a autora se fez presente em momentos distintos no setor de realização dos testes, para observação sistemática e levantamento dos possíveis problemas relacionados à prática.

\section{2a etapa - Capacitação na Unidade}

Logo após o levantamento do diagnóstico situacional, em junho de 2019, foi realizada uma atividade de capacitação, com objetivo de levantar problemas e dúvidas observados pelas técnicas de enfermagem durante o processo de trabalho, além de discussão sobre a temática.

\section{3a etapa - Visita Técnica}

Partindo dos problemas levantados na primeira etapa, a autora sentiu a necessidade de conhecer como os dados registrados eram organizados e armazenados. A mesma realizou uma visita à Coordenação de Área de Planejamento (CAP), no mês de julho de 2019, para melhor entendimento do fluxo das informações após o registro realizado na unidade.

\section{4a etapa - Capacitação pela Secretaria Municipal de Saúde} No mês de setembro de 2019, foi oferecida através da CAP, em parceria com a Secretaria Municipal de Saúde, uma capacitação para realização de testes rápidos, com prática e certificação.

\section{5a etapa - Registro e emissão de laudos}

Notou-se, durante o plano de intervenção, que não eram utilizados os formulários padrão para registro e emissão de laudos para entrega de resultados; os mesmos eram comunicados somente de forma verbal ao usuário. Considerando a importância da utilização do formulário padrão, a autora criou um fluxo e orientou que toda a equipe utilizasse o material já criado e disponível na plataforma SUBPAV, para todas as requisições e liberação de resultados ao usuário.

\section{6a etapa - Elaboração do fluxo}

O fluxo criado a partir dos problemas levantados teve o objetivo de direcionar e implementar um padrão para que todos os profissionais pudessem se nortear, e consequentemente todos os usuários passassem por todas as etapas necessárias.

\section{Relato de Experiência}

Durante o diagnóstico da rotina, observou-se pontos negativos como técnica da execução do teste incorreta e falta de conhecimento teórico, profissionais sem certificação para executar o procedimento e os usuários não recebiam seus resultados nos laudos disponíveis.

Após a implementação do fluxo, notou-se a importância do momento pré e pós testagem, corroborando estudo ${ }^{15}$, que aponta o papel fundamental da enfermagem neste processo.

O profissional de enfermagem capacitado e sensível torna o aconselhamento um processo de escuta ativo, gerando relação de confiança, minimizando dilemas e estressores decorrentes do resultado.

Segundo Araújo ${ }^{10}$, o aconselhamento é umas das principais estratégias de educação em saúde, permitindo uma grande troca entre o aconselhador e o aconselhado.

Segundo estudo ${ }^{15}$, uma das ações mais importantes a serem desenvolvidas pelo enfermeiro no protocolo de testagem é o aconselhamento em seus momentos distintos: pré e pós-testagem. Diante de resultado negativo, o aconselhamento é de suma Importância.

Observou-se também que os usuários passaram a receber seus resultados registrados e liberados no laudo disponível, com todos os dados referentes ao seu teste preenchidos no momento pós teste.

Também como ponto positivo podemos observar que os testes passaram a ser executados na técnica correta, como indicado pelos protocolos, o que influencia diretamente na sua eficácia. Com a criação de um fluxograma, tornou-se mais fácil a identificação visual do processo de trabalho pelos profissionais envolvidos, norteando sua execução.

A orientação do Ministério da Saúde através do Manual Técnico para o diagnóstico da infecção pelo HIV em adultos e crianças ${ }^{14}$ é de que qualquer pessoa pode fazer o teste, desde que seja treinada para tal procedimento. Durante o processo de implementação do fluxo, notou-se que uma técnica de enfermagem não possuía a certificação e realizava tal procedimento. Desta forma, foi possível identificar através do estudo uma falha, que garantiu que a profissional realizasse a capacitação e certificação acerca da temática durante seu turno de trabalho, alcançando-se a meta de certificação de $100 \%$ das profissionais que executam a testagem rápida para IST na unidade.

Outro ponto que vale ressaltar é a estrutura precária da unidade de saúde, o que dificulta o processo de trabalho dentro da sala de procedimentos, não cooperando para a técnica correta de manuseio do material. Por mais que os testes rápidos não requeiram estrutura laboratorial, não há pia para higienização das mãos dentro do setor, faltam materiais de higiene e a refrigeração para o armazenamento dos testes é ineficaz, ou em alguns momentos, ausente. Este foi um problema encontrado que influencia diretamente na eficácia dos testes e, consequentemente, nos seus resultados.

Como resultado, após a criação do fluxo (Figura 1), houve aumento do registro dos testes rápidos nas planilhas, e de forma adequada. 


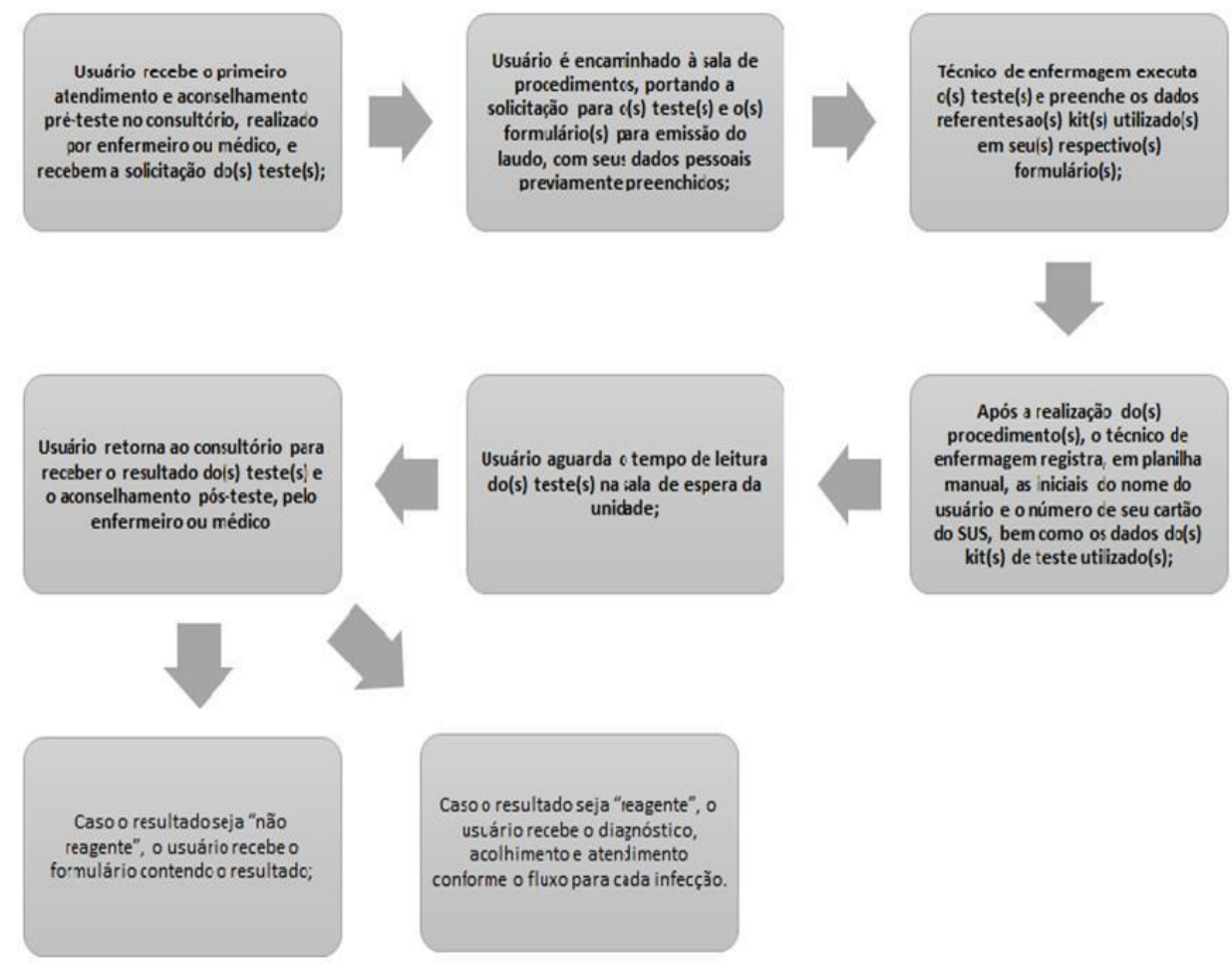

Como Aguiar ${ }^{16: 167}$, os resultados da implementação do fluxo permitem reconhecer que "[...] a realização dos testes rápidos qualifica a atenção básica e proporciona maior resolubilidade e qualidade no atendimento, acolhimento e ações de prevenção e de cuidado à saúde".

\section{Considerações Finais}

Os resultados alcançados por este plano de intervenção podem servir como um disparador para o desenvolvimento de pesquisas acerca da temática, visto que persistem lacunas sobre o tema na literatura científica. Outro ponto levantado por este estudo é que não basta haver disponibilidade de testes, uma vez que não se possua recursos mínimos e uma equipe capacitada para garantir resultados fidedignos.

A dinâmica de trabalho do serviço foi alterada, no sentido de melhorar o processo que envolve a testagem rápida, desde sua solicitação até o registro. Após a implementação do plano, notou-se a melhora na execução, armazenamento e utilização correta dos insumos. A organização de um fluxo é de extrema importância, pois não se limita apenas à sua criação, devendo ser avaliado e rediscutido em equipe com certa frequência, para identificação de novos problemas, observação da persistência de lacunas e ainda de suas potencialidades.

Concluiu-se que a prevalência crescente das ISTs no Brasil e no mundo é um tema que precisa ser discutido dentro das equipes de saúde da família, evidenciando a promoção e prevenção das infecções, considerando que a unidade básica de saúde é a porta principal de entrada dos usuários, devendo assim, oportunizar o acolhimento, aconselhamento, testagem e tratamento das ISTs.

\section{Referências}

1. Pinto MP, et al. Fatores associados às infecções sexualmente transmissíveis: inquérito populacional no município de São Paulo, Brasil. Ciência \& Saúde Coletiva. 2018;23(7):2423-2432. DOI: 10.1590/1413-81232018237.20602016

2. Ministério da Saúde(BR). Secretaria de Vigilância em Saúde. Departamento de Condições Crônicas e Infecções Sexualmente Transmissíveis. Guia de Vigilância em Saúde. [Internet]. Brasília (DF): MS; 2019.[acesso em 05 abr 2021]. Disponível em: https://bvsms.saude.gov.br/bvs/publicacoes/protocolo_clinico_diretrizes_terapeutica_atencao_integral_pessoas_infeccoes_sexualmente transmissiveis.pdf

3. Araújo WJ, Quirino EMB, Pinho CM, Andrade MS. Perception of nurses who perform rapid tests in Health Centers. Rev Bras Enferm [Internet]. 2018;71(Suppl 1):631-6. http://dx.doi.org/10.1590/0034-7167-2017-0298. 
4. Gerstenberger Junior OG, Ribeiro Francisco MT, Bertolossi Marta C, Ribeiro Marques L, Amorim Costa CM, Pimenta de Oliveira MC. O imaginário dos prestadores de serviço do Carnaval sobre prevenção do HIV: uma reflexão psicanalítica. Glob Acad Nurs. 2021; 2 (1):e70. DOI: $10.5935 / 2675-5602.20200070$

5. Rocha KB, et al. Percepção dos Profissionais para Implantação do Teste Rápido para HIV e Sífilis na Rede Cegonha. Brasil. Psicologia e Saúde. 2018;10(3):17-29. http://dx.doi.org/10.20435/pssa.v10i3.555

6. Silva ITS, et al. Cartografia da Implementação do teste rápido anti- HIV na estratégia de saúde da família: perspectiva de enfermeiros. Escola Anna Nery. 2017;21(4): e20170019. DOI: 10.1590/2177-9465-EAN-2017-0019

7. Araujo WJ, et al. Percepção de enfermeiros executores de teste rápido em unidades básicas de saúde. Rev bras enferm. 2018;71(supl1):676-81. DOI: 10.1590/0034-7167-2017-0298>

8. Rocha KB, Ew RAS, Moro LM, Zanardo GLP, Pizzinato A. Aconselhamento na perspectiva de profissionais da atenção básica: desafios na descentralização do teste rápido HIV / Aids. Ciênc Psicol [Internet]. 2018;12(1):67-78. http://dx.doi.org/10.22235/cp.v12i1.1597.

9. Conselho Federal de Enfermagem (COFEN). Parecer de Conselheiro n. 259, 27 de setembro de 2016. Por designação através da Portaria Cofen $n^{\circ} 1285 / 2016$, recebemos o Processo Administrativo $n^{\circ}$ 0623/2016, que tem por objeto analise da solicitação de revogação do Parecer Normativo 001/2013, para emissão de parecer a ser apreciado pelo pleno desta casa [Internet]. Brasília (DF): COFEN; 2016 [acesso em 05 abr 2021]. Disponível em: http://www.cofen.gov.br/parecer-de-conselheiro-n-2592016_46252.html

10. Araujo GM, Nardino LJ, Resdorfer N, Begninin D. Aconselhamento pré-testagem rápida: uma proposta de educação em saúde. Revista Espaço Ciência e Saúde.2017;5(1)

11. Ew RAS, Ferreira GS, Moro LM, Rocha KB. Estigma e teste rápido na atenção básica: percepção de usuários e profissionais. Rev Bras Promoç Saúde [Internet]. 2018;31(3): 1-11. https://doi.org/10.5020/18061230.2018.7463

12. Conselho Federal de Enfermagem (COFEN). Resolução n.o 544, de 18 de maio de 2017. Art. 1o Revogar a Resolução Cofen no 159/1993, que dispõe sobre a consulta de Enfermagem, assinada no dia 19 de abril de 1993, data em que entrou em vigor [Internet]. Brasília (DF): COFEN; 2017 [acesso em 05 abr 2021] Disponível em: http://www.cofen.gov.br/resolucao-cofen-no-05442017_52029.html

13. Robbins SP. Administração: mudanças e perspectivas. 7. ed. São Paulo: Saraiva; 2007.

14. Ministério da Saúde (BR). Secretaria de Vigilância em Saúde. Departamento de Vigilância, Prevenção e Controle das Infecções Sexualmente Transmissíveis, do HIV/Aids e das Hepatites Virais. Manual Técnico para o Diagnóstico da Infecção pelo HIV em Adultos e Crianças. [Internet]. Brasília (DF): MS, 2016 [acesso em 05 abr 2021]. Disponível em: https://bvsms.saude.gov.br/bvs/publicacoes/manual_tecnico_diagnostico_infeccao_hiv.pdf

15. Silva $\mathrm{O}$, Tavares LHL, Paz LC. As atuações do enfermeiro relacionadas ao teste rápido anti-HIV diagnóstico: uma reflexão de interesse da enfermagem e da saúde pública. Revista Enfermagem em foco. 2011;2(supl):58-62.

16. Aguiar DS, et al. Enfermagem frente à testagem rápida de sífilis, HIV e hepatites virais em uma comunidade periférica de Macapá, Amapá. Braz. J. Hea. Rev., Curitiba. 2018;1(1):164-184. 